\title{
Treatment of Clostridium Difficile Infection in Community Teaching Hospital: A Retrospective Study
}

\author{
Ali Elbeddini \\ Department of Clinical Pharmacy, Winchester District Memorial Hospital WDMH, Winchester, Canada \\ Email address: \\ aelbeddini@wdmh.on.ca \\ To cite this article: \\ Ali Elbeddini. Treatment of Clostridium Difficile Infection in Community Teaching Hospital: A Retrospective Study. International Journal \\ of Infectious Diseases and Therapy. Vol. 3, No. 3, 2018, pp. 52-61. doi: 10.11648/j.ijidt.20180303.12
}

Received: September 14, 2018; Accepted: October 9, 2018; Published: October 23, 2018

\begin{abstract}
Clostridium difficile infection (CDI) is responsible for $15-25 \%$ cases of health-care associated diarrhea. The CDI treatment algorithm used at our hospital is adapted from the Infectious Diseases Society of America 2010 C. difficile guideline. The primary objective of this study was to assess the treatment adherence to our algorithm; this was defined as therapy consisting of the appropriate antibiotic, dose, route, interval and duration indicated based on the disease severity and episode within 24 hours of diagnosis. In addition, our study also described the population and their risk factors for CDI at our hospital. This was a single-centre, retrospective cohort chart review of CDI cases that were diagnosed at admission or during hospitalization from June 1st 2017 to June 30th 2018. Sixty cases were included, of which adherence to our algorithm was $50 \%$. Overall, severe CDI had the highest treatment non-adherence $(83 \%)$ and the biggest contributing factor was prescribing the wrong antibiotic (72\%). In severe CDI, which warrants vancomycin monotherapy, wrong antibiotic consisted of metronidazole monotherapy $(55 \%)$ or dual therapy with metronidazole and vancomycin (45\%). Patients were mostly older, females being treated for an initial episode of mild to moderate CDI. Common risk factors identified were age over 65 years $(80 \%)$, use of antibiotics (83\%) and proton pump inhibitors (PPI) $(68 \%)$ within the previous three months. The use of a PPI in this study, a modifiable risk factor without a clear indication was $35 \%$. The conclusion was that there is an area for antimicrobial stewardship intervention in CDI treatment at our hospital is prescribing the right antibiotic based on the CDI indication. In severe CDI, an emphasis should be on prescribing vancomycin monotherapy as the drug of choice. PPI use should be reassessed for tapering when appropriate.
\end{abstract}

Keywords: Clostridium Difficile Infection, Adherence, Infectious Diseases Society of America 2010 C. Difficile Guideline

\section{Introduction}

\subsection{Background}

C. difficile is an anaerobic, spore producing, gram-positive bacterium that is transmitted by the fecal-oral route. [1] Since September 2008, Clostridium difficile infections (CDI) are one of several monthly patient safety indicators reportable to the Ontario Ministry of Health and Long-Term Care (MOHLTC) under the Public Hospitals Act. [2] From June 1 st 2017 to June $30^{\text {th }} 2018$, a minimum of 50 CDI cases, including both initial and recurrent cases were reported at WDMH. Recurrent CDI is defined as another episode of CDI which develops within eight weeks after the onset of a previous episode that resolved with appropriate treatment. [3, 15]. the rate of recurrence after an initial episode of CDI is 6
$-25 \%$ and increases with recurrent episodes of CDI. [1, 4] It can be manifested either by another infection caused by the original strain of $\mathrm{C}$. difficile or a new infection caused by a new strain of $\mathrm{C}$. difficile while the microflora in the colon is returning to normal levels. [1]

Hospitals are associated with a higher risk of transmission due to environmental contamination, the frequent use of antibiotics, the presence of other CDI cases and poor hand hygiene practices. The use of antibiotics disrupts the microflora of the colon allowing $\mathrm{C}$. difficile to grow in high concentrations. [5] As a result, CDI rates tend to be higher between the months of November to March due to increased respiratory infections requiring use of antibiotics. [2]

Although all antibiotics can contribute to CDI, the use of clindamycin, third-generation cephalosporin and fluoroquinolone are associated with the highest rates of CDI. 
[5] Other medications associated with an increased risk of CDI include gastric acid suppressing agents such as proton pump inhibitors (PPIs) and histamine-2 receptor antagonists (H2RAs), promoting an environment suitable for C. difficile bacteria to survive and are associated with an increased risk of CDI. [5] Immunosuppressing medications such as the use of antineoplastics or steroids decrease the immune system's ability to produce antibodies. [2] Other risk factors associated with an increased exposure to C. difficile and antibiotic use include gastrointestinal surgery, irritable bowel disease, diabetes, cardiovascular, respiratory or kidney disease, patients over 65 year old, use of nasogastric tubes, prolonged hospitalization or exposure to long term care facilities. [3, 6]

Common symptoms of CDI include watery diarrhea, nausea, abdominal pain and fever. [1] If left untreated, CDI can, in rare instances, result in pseudomembranous colitis, toxic megacolon and death. [2] Therefore, minimizing prolonged durations of antibiotic therapy and antibiotic use without appropriate indications, and switching from intravenous to oral therapy when possible to shorten hospital stay will help decrease the risk of developing CDI. [5] Similarly, appropriate treatment of CDI based on the severity of the infection will help decrease the risk of developing a recurrent CDI. [5]

Sixty cases were included, of which adherence to our algorithm was 50\%. Overall, severe CDI had the highest treatment non-adherence $(83 \%)$ and the biggest contributing factor was prescribing the wrong antibiotic (72\%). In severe CDI, which warrants vancomycin monotherapy, wrong antibiotic consisted of metronidazole monotherapy (55\%) or dual therapy with metronidazole and vancomycin (45\%). Patients were mostly older, females being treated for an initial episode of mild to moderate CDI. Common risk factors identified were age over 65 years $(80 \%)$, use of antibiotics $(83 \%)$ and proton pump inhibitors (PPI) $(68 \%)$ within the previous three months. The use of a PPI in this study, a modifiable risk factor without a clear indication was 35\%. The conclusion was that there is an area for antimicrobial stewardship intervention in CDI treatment at our hospital is prescribing the right antibiotic based on the CDI indication. In severe CDI, an emphasis should be on prescribing vancomycin monotherapy as the drug of choice. PPI use should be reassessed for tapering when appropriate.

At WDMH, CDI is diagnosed by a positive stool detection of both C. difficile antigen and C. difficile toxin A and B. If only one of the two is positive, a positive polymerase chain reaction (PCR) test for $\mathrm{C}$. difficile toxin $\mathrm{B}$ is required to confirm diagnosis. [7] Contrary, if the detection is negative for both antigen and toxins, or if the PCR test is negative, no CDI diagnosis is made. [7] In addition, the passage of three or more unformed stools defined by Bristol stool type $6-7$ (See Table 1) in 24 hours must also be present in order to confirm the diagnosis of CDI, except patients diagnosed with an ileus or toxic megacolon. [3] prior to initiating treatment in symptomatic patients with positive laboratory findings, the classification of the severity of CDI must be established (See Table 2). To distinguish between severities, other additional signs and symptoms must be considered. Mild and moderate $\mathrm{CDI}$ is defined as having signs and symptoms including fever, increased abdominal pain, signs of dehydration such as decreased urine output, and leukocytosis with a WBC $<15000$ cells/ $\mu$ L. [8] Severe CDI is defined as having signs and symptoms including fever, severe abdominal pain, signs of sepsis such as confusion, tachycardia and decreased urine output, acute renal dysfunction (defined as an elevated serum creatinine greater than 1.5 times the premorbid level) and leukocytosis with $\mathrm{WBC} \geq 20000$ cells/ $\mu \mathrm{L}$. [8, 9] Similarly, severe and complicated CDI is defined as having symptoms of severe CDI in addition to either having an ileus, toxic megacolon, shock such as a drop in systolic blood pressure. [8]

Table 1. Bristol Stool Chart.

\begin{tabular}{|c|c|}
\hline Type 1 & $\begin{array}{l}\text { Type 1: Stools appear in separate, hard lumps, similar to nuts. Type } 1 \text { stools remained } \\
\text { in the colon the longest amount of time; a sure sign you're constipated; most common } \\
\text { stools. }\end{array}$ \\
\hline Type 2 & $\begin{array}{l}\text { Stools are sausage-like in appearance but lumpy. Indicate toxic constipation and need for } \\
\text { intestinal cleansing }\end{array}$ \\
\hline Type 3 (Normal) & Stools come out similar to a sausage but with cracks in the surface. \\
\hline Type 4 (Normal) & Stools are smooth and soft in the form of a sausage or snake. \\
\hline Type 5 & $\begin{array}{l}\text { Stools form soft blobs with clear-cut edges, and easily pass through the digestive system. } \\
\text { Soft diarrhea, it may indicate a possible risk for bowel disease; also indicate you are toxic } \\
\text { and need regular intestinal cleansing. }\end{array}$ \\
\hline Type 6 & $\begin{array}{l}\text { Stools have fluffy pieces with ragged edges. Considered mushy stools, they indicate } \\
\text { diarrhea; and that you are toxic and need regular intestinal cleansing. }\end{array}$ \\
\hline Type 7 & $\begin{array}{l}\text { Stool is mostly liquid with no solid pieces. Passed quickly through the colon; is indicative } \\
\text { of severe diarrhea possibly as a result of a viral or bacterial infection. See a doctor as soon } \\
\text { as possible. }\end{array}$ \\
\hline
\end{tabular}


Table 2. Classification of Disease Severity.

\begin{tabular}{ll}
\hline Severity & Signs and symptoms \\
\hline \multirow{2}{*}{ Mild to Moderate } & WBC $<20000$ cells $/ \mu \mathrm{L}$ \\
& $\mathrm{SCr}<1.5$ times the premorbid level \\
Severe & $\mathrm{WBC} \geq 20000$ cells $/ \mu \mathrm{L}$ \\
& $\mathrm{SCr} \geq 1.5$ times the premorbid level \\
Severe to Complicated & Hypotension \\
& Ileus \\
\hline
\end{tabular}

\subsection{Guidelines Recommendations}

There are three guidelines available for the treatment of C. difficile: The Infectious Diseases Society of America (IDSA) published in 2010, the American College of Gastroenterology (ACG) published in 2013, and the European Society of Clinical Microbiology and Infectious Diseases (ESCMID) published in 2014. All three guidelines recommend similar therapies for different treatments of CDI with minor differences such as prolonging duration of therapy and adding alternative therapies to their recommendations (See Table 3). [1, 3, 11]

Table 3. Current Antibiotic Treatment Recommendations for CDI [1, 10].

\begin{tabular}{|c|c|c|c|c|}
\hline Level of severity & IDSA (2010) & ACG (2013) & ESCMID (2014) & WDMH (2017) \\
\hline \multicolumn{5}{|l|}{ Initial episode of CDI } \\
\hline Mild to Moderate & $\begin{array}{l}\text { Metronidazole } 500 \mathrm{mg} \text { po } \\
\text { q8h X 10-14 days }\end{array}$ & $\begin{array}{l}\text { Metronidazole } 500 \mathrm{mg} \text { po } \\
\text { q } 8 \mathrm{~h} \text { X } 10 \text { days (If no } \\
\text { improvement in } 5-7 \text { days, } \\
\text { consider change to severe } \\
\text { CDI treatment) }\end{array}$ & $\begin{array}{l}\text { Metronidazole } 500 \mathrm{mg} \text { po } \\
\text { q8h X } 10 \text { days }\end{array}$ & $\begin{array}{l}\text { Metronidazole } 500 \mathrm{mg} \text { po } \\
\text { q } 8 \mathrm{~h} \text { X } 10-14 \text { days(If no } \\
\text { improvement in } 5 \text { days or } \\
\text { clinical worsening, change } \\
\text { to CDI severe treatment) }\end{array}$ \\
\hline Severe & $\begin{array}{l}\text { Vancomycin } 500 \mathrm{mg} \text { po q6h } \\
\text { and metronidazole } 500 \mathrm{mg} \\
\text { iv q } 8 \mathrm{~h} \text { and(If ileus present: } \\
\text { add vancomycin } 500 \mathrm{mg} \text { in } \\
100 \mathrm{mLNS} \text { pr q6h) }\end{array}$ & $\begin{array}{l}\text { Vancomycin } 125 \mathrm{mg} \text { po q6h } \\
\text { X } 10 \text { days }\end{array}$ & Vancomycin $125 \mathrm{mg}$ po q6h & $\begin{array}{l}\text { Vancomycin } 125 \mathrm{mg} \text { po q6h } \\
\text { X } 10-14 \text { days (If no } \\
\text { response or symptoms } \\
\text { worsening, consult Internal } \\
\text { Medicine) }\end{array}$ \\
\hline Severe/complicated & $\begin{array}{l}\text { Vancomycin } 500 \mathrm{mg} \text { po q6h } \\
\text { and metronidazole } 500 \mathrm{mg} \\
\text { iv q8h and(If ileus present: } \\
\text { add vancomycin } 500 \mathrm{mg} \text { in } \\
100 \mathrm{~mL} \text { NS pr q6h) }\end{array}$ & $\begin{array}{l}\text { Vancomycin } 125 \mathrm{mg} \text { po } \\
\mathrm{q} 6 \mathrm{~h}, 500 \mathrm{mg} \text { in } 500 \mathrm{~mL} \\
\text { saline as enema pr q6h and } \\
\text { metronidazole } 500 \mathrm{mg} \text { iv } \\
\mathrm{q} 8 \mathrm{~h}\end{array}$ & X 10 days & $\begin{array}{l}\text { Vancomycin } 125-500 \mathrm{mg} \text { po } \\
\text { q6h and metronidazole } 500 \\
\mathrm{mg} \text { iv q } 8 \mathrm{~h} \text { (Consider } \\
\text { vancomycin pr or } \\
\text { immunoglobulin iv) }\end{array}$ \\
\hline $2^{\text {nd }}$ recurrent episode of CDI & $\begin{array}{l}\text { Vancomycin in a tapered } \\
\text { and/or }\end{array}$ & vancomycin pulse regimen & $\begin{array}{l}\text { Fecal transplant combined } \\
\text { with oral antibiotic }\end{array}$ & $\begin{array}{l}\text { Vancomycin } 125 \mathrm{mg} \text { po } \mathrm{q} 6 \mathrm{~h} \\
\mathrm{X} 10-14 \text { days }\end{array}$ \\
\hline $\begin{array}{l}\text { Pregnant patients or } \\
\text { Intolerant to Metronidazole }\end{array}$ & No recommendation & $\begin{array}{l}\text { Vancomycin } 125 \mathrm{mg} \text { po } \mathrm{q} 6 \mathrm{~h} \\
\text { X } 10 \text { days }\end{array}$ & No recommendation & $\begin{array}{l}\text { Same treatment as severe } \\
\text { CDI }\end{array}$ \\
\hline
\end{tabular}

For treatment of initial episodes of mild to moderate CDI, the general consensus from all three guidelines is metronidazole $500 \mathrm{mg}$ po $\mathrm{q} 8 \mathrm{~h}$ for 10 to 14 days. $[1,3,11]$ The ESCMID guidelines also recommend vancomycin 125 $\mathrm{mg}$ po q6h for 10 days as an alternative to metronidazole. [3] The ACG recommends switching therapy to treatment for severe CDI if there is no improvement after five to seven days with the current therapy. [11]

For treatment of initial episodes of severe CDI, the general consensus from all three guidelines is vancomycin $125 \mathrm{mg}$ po q6h for 10 to 14 days. [1, 3, 11] Another alternative the ESCMID recommends is to consider increasing the vancomycin dose to $500 \mathrm{mg} \mathrm{q} 6 \mathrm{~h}$ for 10 days. [3] For treatment of initial episodes of severe, complicated CDI, the general consensus from all three guidelines is vancomycin 125 - $500 \mathrm{mg}$ po q6h \pm metronidazole $500 \mathrm{mg}$ IV q $8 \mathrm{~h}$.

In $[1,3,11]$ the presence of an ileus, the IDSA recommends adding vancomycin $500 \mathrm{mg}$ in approximately $100 \mathrm{~mL}$ normal saline pr $\mathrm{q} 6 \mathrm{~h}$ as a retention enema. [1] In an ileus, toxic megacolon or abdominal distension, the ACG recommends triple therapy consisting of vancomycin $500 \mathrm{mg}$ in a volume of $500 \mathrm{~mL}$ pr q6h, vancomycin $500 \mathrm{mg}$ po q6h and standard iv metronidazole therapy. [11]

For the first recurrent episode of CDI, all the guidelines recommend following the same treatment as for an initial episode of CDI. $[1,3,11]$ In the second or multiple recurrent episodes of CDI, the general consensus to avoid peripheral neuropathy with metronidazole, is vancomycin $125 \mathrm{mg}$ po q6h for 10 to 14 days followed by either a pulse or taper regimen. $[1,3,11]$ The intermittent dosing that follows the scheduled vancomycin dosing continues to suppress levels of $\mathrm{C}$ difficile while allowing the microflora of the colon to return to normal. [11]

It is recommended to avoid metronidazole in pregnant women who are in their first trimester since it has been associated with facial anomalies. As a result, the ACG recommends standard vancomycin therapy for 10 days in this population. [11] The IDSA and ESCMID do not have specific 
treatment recommendations for this population.

In addition to the pharmacological treatment for CDI, nonpharmacological options such as the use of fecal transplant or surgery may be indicated in specific cases of recurrent CDI. [12] Incorporation of infection control and prevention techniques such as proper hand hygiene, environmental disinfection and single room isolation will also help minimize the spread of $\mathrm{C}$. difficult. [12] The role of probiotics is still uncertain in the prevention or treatment of CDI and is currently not part of the treatment algorithm.[1] Finally, other pharmacological treatment approaches to increase the efficacy of treatment involves discontinuing current laxatives and acid suppressing medications, if possible since they worsen symptoms of CDI and increase the risk of recurrent CDI, respectively.[10] The IDSA and ACG guidelines also recommend avoiding antidiarrheal medications since they increase the retention of $\mathrm{C}$. difficile toxins and The risk for toxic megacolon. $[4,8,11]$

\subsection{Current Situation}

At WDMH, the C. difficile treatment algorithm is adapted from the The Ottawa Hospital (TOH)

C. difficile treatment algorithm (See Table 3). Both hospital algorithms are based on the IDSA 2010 guidelines. For this research project, the two main antibiotics included will be metronidazole and vancomycin since the new antibiotic, fidaxomicin and fecal transplant are not included in the WDMH

Treatment algorithm.

For treatment of initial episodes of mild to moderate CDI, the WDMH algorithm recommends metronidazole $500 \mathrm{mg}$ po $\mathrm{q} 8 \mathrm{~h}$ for 10 to 14 days. [10] If there is no improvement or if there is significant clinical deterioration at Day 5, therapy should be escalated for treatment of severe CDI, which is vancomycin $125 \mathrm{mg}$ po $\mathrm{q} 6 \mathrm{~h}$ for 10 to 14 days. [10] In the presence of ileus or toxic megacolon, dual therapy of vancomycin $125-500 \mathrm{mg}$ po $\mathrm{q} 6 \mathrm{~h}$ and standard iv metronidazole therapy with the consideration of administering vancomycin $500 \mathrm{mg}$ in the form of an enema in $100 \mathrm{~mL}$ normal saline (NS) for 60 minutes $\mathrm{q} 4-12 \mathrm{~h}$ or intravenous immunoglobulin is recommended. [10] Treatment for first recurrent episode of CDI remains the same as initial episode of CDI. Treatment for second or multiple recurrent episodes of CDI is vancomycin $125 \mathrm{mg}$ po q6h for 10 to 14 days followed by either a pulse or taper regimen. [3, 10] In patients who are pregnant or who are intolerant to metronidazole, the WDMH algorithm recommends vancomycin $125 \mathrm{mg}$ po q6h for 10 to 14 days. [10]

\subsection{Extending Duration of CDI Treatment with Concomitant Antibiotics}

Patients who receive antibiotics for other infections during treatment of CDI or when the colon microflora has not returned to normal are at an increased risk of recurrent CDI. Since the disruption of the colon microflora can lasts for days and up to weeks after completion of therapy, some clinicians continue treatment of CDI until the antibiotic therapy is completed. [1] However, it is unknown whether this practice reduces the risk of a recurrent CDI. [1] Contrary, the ACG guidelines state that there is no evidence to support the continuation of CDI treatment in patients who are also on non-CDI antibiotics. [11] Therefore, the evidence and consensus regarding the duration of $\mathrm{C}$. difficile therapy is lacking. There is however, expert opinion-based recommendations that in patients receiving concurrent antibiotics for other infections, treatment for C. difficile should be continued for at least seven days after the completion of non-CDI antibiotics. [13] Depending on the number of episodes, the duration of extended CDI therapy may be adjusted to correlate with the associated risk of recurrence. As a result, at WDMH, although it is not part of the treatment algorithm, it is recommended to continue $\mathrm{C}$. difficile treatment for a minimum of seven days in patients who have completed non-CDI antibiotics. [10]

\subsection{Primary Research Objectives}

The first primary objective of this study is to describe the CDI treatment adherence at WDMH to the WDMH C. difficile treatment algorithm, which is based on the IDSA 2010 C. difficile guidelines. Adherence to treatment algorithm is defined as the appropriate antibiotic, dose, route, interval, duration, time to start and stop dates of antibiotics indicated based on the classification of CDI severity. The second primary objective is to describe the population and their risk factors for CDI at WDMH.

\subsection{Secondary Research Objectives}

Describe the current practice at WDMH regarding continuation of CDI treatment after completion of non-CDI antibiotics used to treat other infections.

Describe the rate of recurrent CDI associated with the duration of CDI therapy after the completion of non-CDI antibiotics used to treat other infections.

The purpose of this study was to assess the CDI treatment adherence to our algorithm to help identify areas for antimicrobial stewardship interventions.

\section{Methodology}

\subsection{Study Type}

This research project was a single-centre, retrospective cohort study.

Study Sample and Inclusion Criteria:

All patients were eligible if they were diagnosed with an initial or recurrent episode of CDI (defined as a previous episode of CDI that developed in the previous eight weeks and was resolved with appropriate treatment) at admission or during their hospitalization at WDMH from June 1, 2017 to June 30th, 2018. CDI cases were defined as a diagnosis of CDI that consisted of a positive stool test for $\mathrm{C}$. difficile antigen, toxin $\mathrm{A}$ and $\mathrm{B}$ (or positive PCR test for toxin $\mathrm{B}$ if necessary when stool toxin is not positive) along with 
passage of three or more unformed stools, defined as Bristol Scale Type $6-7$ (See Table 1) in 24 hours. Alternatively, a colonoscopy detecting pseudomembranous colitis is another form of diagnosis. Treatment adherence was based on he CDI treatment initiated and disease severity listed in the algorithm including hypotension defined as SBP $<90 \mathrm{mmHg}$ and measured white blood cell (WBC) count within the 24 hours of diagnosis.

No sample size calculation was defined since this was a retrospective cohort study with chart reviews. The sample size referred to the number of CDI cases and not the number of CDI patients. Therefore, the minimum sample size of 50 CDI cases was chosen due to the convenience of data available from June 1, 2017 to June 30th, 2018, which was reasonable due to the descriptive purpose of this study. The maximum sample size chosen was $100 \mathrm{CDI}$ cases due to time limitation factor to complete this project during one year. Therefore, this sample size range was enough to provide reliable descriptive statistics that can also be used for areas of improvement.

\subsection{Data Collection}

Two different sources were used to collect data and they included QuadraMed computer program at WDMH and patient records. Quality control for $10 \%$ of the CDI cases were reviewed by Primary investigator.

\subsection{Data Analysis}

All data collected were entered and analysed with Microsoft Excel. The first primary objective, description of treatment adherence to our algorithm, based on the IDSA 2010 C. difficile guidelines was expressed as nominal data as a percentage of yes or no. The analyses of breakdown of different components were based on the disease severity. If the wrong drug were prescribed, that would automatically be counted, as non-adherent and no further analyses would be done. If a regimen contained the right drug, further investigation regarding appropriate dose, route, interval and duration were analyzed. The second primary objective, description of population and their risk factors were expressed using descriptive statistics including the calculation of the mean and median, as applicable. The first secondary objective, continuation of CDI treatment after completion of non-CDI antibiotics, was expressed as continuous data based on the number of days therapy was continued. The second secondary objective, describing the association between the rates of recurrent CDI and duration of CDI therapy after the completion of non-CDI antibiotics was expressed as nominal data as a percentage of yes or no categorized by the days of CDI therapy was continued into either less than seven days or seven days or more.

Overall outcomes including CDI resolution defined as no loose stool (Bristol Type $6-7$ ) within 48 hours prior to discharge, 30-day all-cause mortality, 30-day readmission and 8-week CDI recurrence were analyzed based on receiving adherent and non-adherent treatment and were reported using the odds ratio test. Statistical significance was defined as having a $\mathrm{p}$-value $<0.05$.

\section{Results}

Of the 75 cases identified, 60 cases met the inclusion criteria. The total of 60 cases were based on 56 patients as two patients had two episodes of CDI each and were accounted twice (Figure 1). The reasons cases were excluded were the following: four cases had a negative stool test result and no colonoscopy done, four cases had a negative PCR test and no colonoscopy, two cases did not receive treatment for $\mathrm{CDI}$, two cases identified in the emergency room and were not admitted, two cases were already discharged by the time the results came back, and one case was on a vancomycin taper initiated from another institution.

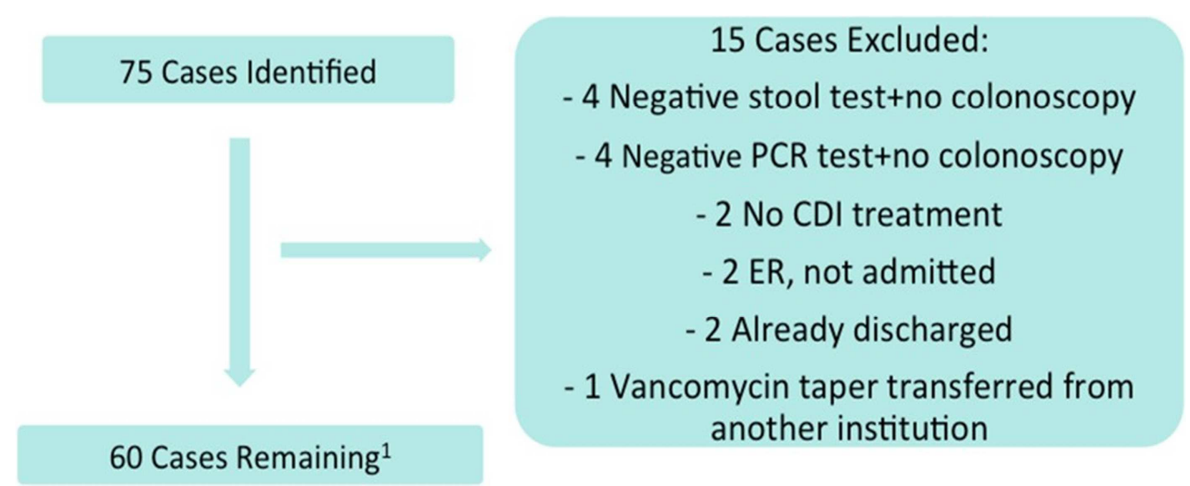

Figure 1. Flow diagram of case selection.

The demographics of our patient population had a mean age of $75 \pm 15$ years and a mean body mean index (BMI) of $21 \pm 14 \mathrm{~kg} / \mathrm{m}^{2}$. The average length of stay for one admission (LOS) was $12 \pm 24$ days. The gender breakdown in the study consisted of 38 females and 22 males. The breakdown of CDI episodes was the following: 55 cases were initial CDI cases, 4 cases were first CDI recurrences, and one case was a second CDI recurrence (Table 4). Based on the disease severity that patients presented within 24 hours of CDI diagnosis, 34 cases were classified as mild to moderate, 24 cases were classified as severe, and two cases were classified as severe and complicated. 
Table 4. Baseline Characteristics.

\begin{tabular}{ll}
\hline & Average \pm SD \\
\hline Age (years & $75 \pm 15$ \\
Body Mass Index (kg/m2) & $21 \pm 14$ \\
Length of stay (days) & $12 \pm 24$ \\
& $\#$ out of $60(\%)$ \\
Female & $38(63)$ \\
Episode:Initial & $55(92)$ \\
1st recurrence & $4(7)$ \\
2nd recurrence & $1(2)$ \\
Severity & \\
Mild to Moderate & $34(57)$ \\
Severe & $24(40)$ \\
Severe to complicated & $2(3)$ \\
\hline
\end{tabular}

The following risk factors were identified: use of at least one antibiotic defined as use of at least one dose in the previous three months prior to diagnosis occurred in 50 cases, of which the breakdown of the three antibiotics most associated with CDI were the following: fluoroquinolones use in 30 cases, third generation cephalosporin in 20 cases, and clindamycin in two cases. Age over 65 years represented 48 cases. The use of acid reducing agents defined as use of at least one dose in the previous three months prior to diagnosis of a proton pump inhibitor (PPI) or histamine-2 receptor antagonist occurred in 41 cases and 5 cases, respectively. Previous hospitalization at the WDMH occurred in 23 cases and length of stay equal or more than two weeks prior to CDI diagnosis occurred in 11 cases. Finally, recurrent CDI, which was defined as having an episode of CDI in the previous eight weeks occurred in five cases (Table 5).

Table 5. Risk factors.

\begin{tabular}{ll}
\hline & \# out of 60 (\%) \\
\hline Age more than $>$ 65 years old & $48(80)$ \\
Length of stay more than 14 days & $11(18)$ \\
Clindamycin & $2(3)$ \\
Fluoroquinolone & $30(50)$ \\
$3^{\text {rd }}$ generation cephalosporin & $20(33)$ \\
At least one Antibiotic & $50(83)$ \\
Proton pump inhibitor & $41(68)$ \\
Histamine 2 Receptor Antagonist & $5(8)$ \\
Hospitalization & $23(38)$ \\
Recurrent CDI & $5(8)$ \\
\hline
\end{tabular}

The adherence to our CDI treatment algorithm occurred in 30 cases out of a total of 60 cases included in this study (Figure 2). The breakdown of the CDI severity showed adherence in 26 cases out of a total of 35 mild to moderate cases, 4 cases out of a total 23 severe cases, and zero cases out of a total of two severe and complicated cases.

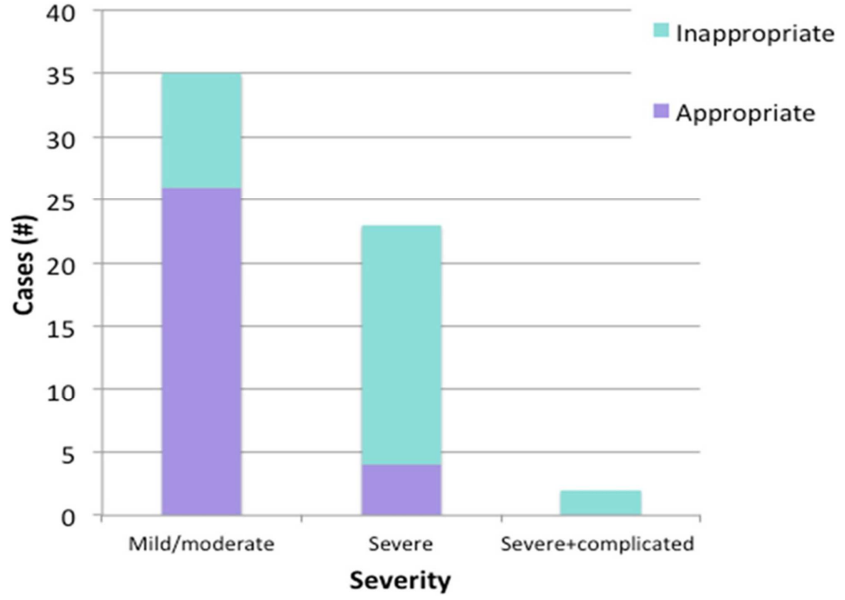

Figure 2. Treatment Appropriateness by Severity.

The different components contributing to treatment nonadherence occurred in 40 antibiotic regimens prescribed from 30 non-adherent cases (Figure 3). Twenty-nine antibiotic regimens were non-adherent due to the wrong drug being prescribed. The remaining non-adherence occurred in 11 correctly prescribed drug regimens, which four had the wrong dose, four had the wrong route and three had the wrong duration. No non-adherent intervals were noted.

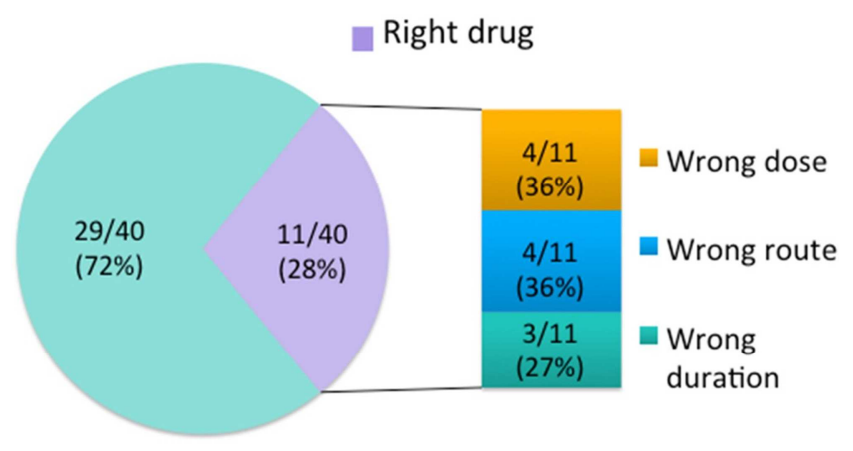

Wrong drug

Figure 3. Components of Inappropriate Treatment.

Wrong drugs were prescribed in 29 cases (Figure 4). Based on the disease severity, the breakdown for different regimens 
of inappropriate drugs were as follows: in the mild to moderate group, where the algorithm recommends treatment with metronidazole monotherapy, three cases out of seven were prescribed vancomycin monotherapy while the four remaining cases were prescribed a combination of metronidazole and vancomycin. In the severe group, where the algorithm recommends treatment with vancomycin monotherapy, 11 out of 20 were prescribed metronidazole monotherapy and the remaining nine cases were prescribed a combination of metronidazole and vancomycin. In the severe and complicated group, where the algorithm recommends treatment with metronidazole and vancomycin, all two cases were prescribed with metronidazole monotherapy.

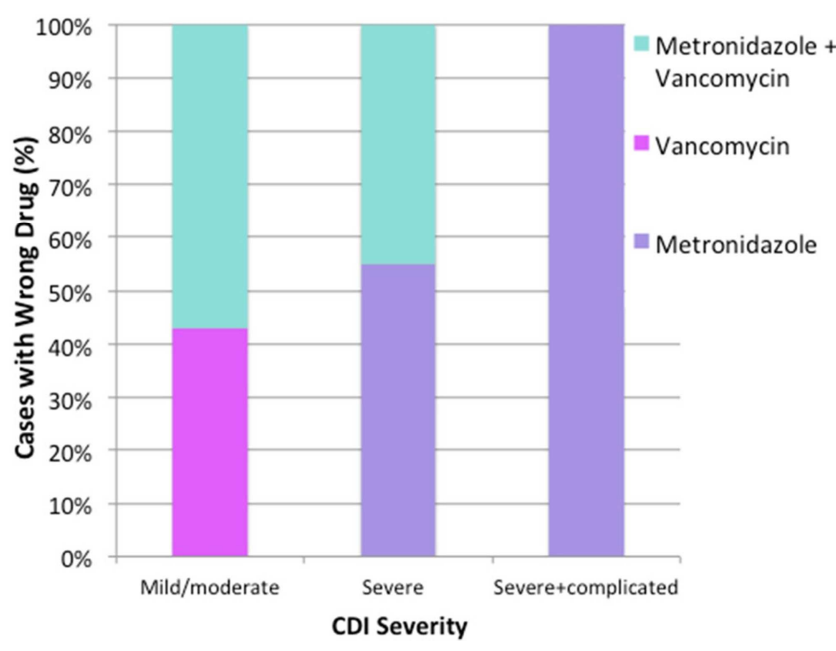

Figure 4. Components of Wrong Drug by Severity.

There was a total of seven cases where CDI antibiotics were continued past the use of non-CDI antibiotics used to treat other infections (Figure 5). Continuation ranged from three days up to 15 days with a mean and median of seven days. Out of the seven cases of continuing CDI antibiotics, three recurrent CDIs were identified.

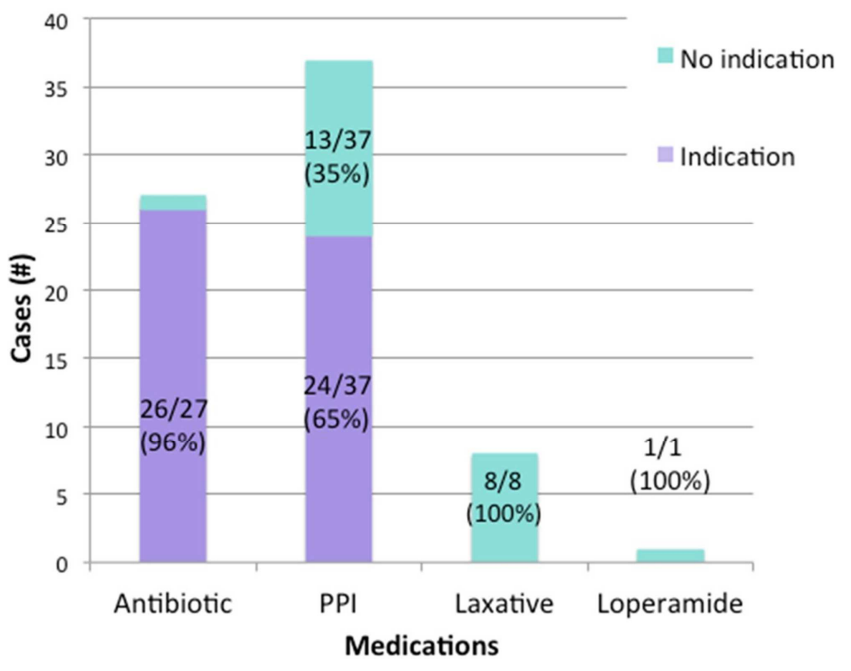

Figure 5. Indications for Medications Used During CDI.

Overall outcomes (See table 6) measured included CDI resolution defined as no loose stool (Bristol Scale Type $6-7$ ) for at least 48 hours prior to discharge, 30-day all-cause mortality, 30-day readmission and CDI recurrence defined as another episode within eight weeks (Table 6). CDI resolution occurred in 35 cases, of which 11 cases were non-adherent and the remaining 24 cases were adherent (non-adherent vs. adherent was $31 \%$ vs. $69 \%$, odds ratio $(\mathrm{OR})=6.91,95 \%$ confidence interval $(\mathrm{CI})=2.16-22.10$, $\mathrm{p}$-value $=0.001) .30$ day all-cause mortality occurred in 16 cases, of which 12 cases were non-adherent and the remaining two cases were adherent (non-adherent vs. adherent was $86 \%$ vs. $14 \%$, OR $=$ 9.33, 95\% CI $=1.97-46.69, \mathrm{p}$-value $=0.007)$. There were 37 possible cases of 30-day remission outcomes, of which there were eight cases that had 30-day remission. Three of the eight cases were non-adherent and the remaining five cases were adherent (non-adherent vs. adherent was $37 \%$ vs. $63 \%, \mathrm{OR}=0.88,95 \% \mathrm{CI}=0.18-4.24, \mathrm{p}$-value $=0.874)$. For recurrent $\mathrm{CDI}$, there were 39 possible cases, of which seven cases had recurrent CDI. Three of seven cases were nonadherent, while the remaining four cases were adherent (nonadherent vs. adherent was $43 \%$ vs. $57 \%$, OR $=97,95 \% \mathrm{CI}=$ $0.19-4.93, \mathrm{p}$-value $=0.971)$.

Table 6. Adherent treatment and overall outcomes.

\begin{tabular}{|c|c|c|c|c|c|c|}
\hline Outcomes & Incidence $(\%)$ & Inappropriate treatment $(\%)$ & Appropriate treatment (\%) & OR & $95 \%$ CL & P-value \\
\hline CDI resolution (60 cases) & $35 / 60(58)$ & $11 / 35(31)$ & $24 / 35(69)$ & 6.91 & $2.16-22.10$ & 0.001 \\
\hline 30 day- all cause mortality( 60 cases $)$ & $14 / 60(23)$ & $12 / 14(86)$ & $2 / 14(14)$ & 9.33 & $1.87-46.69$ & 0.007 \\
\hline 30 day readmission (37 cases) & $8 / 37(22)$ & $3 / 8(37)$ & $5 / 8(63)$ & 0.88 & $0.18-4.24$ & 0.874 \\
\hline
\end{tabular}

The use of non-CDI antibiotic during CDI treatment occurred in 27 cases, of which 26 cases had an indication and the remaining case had no indication. The use of PPI during CDI treatment occurred in 37 cases, of which 24 cases had an indication and the remaining 13 cases did not. The use of ongoing laxatives occurred in eight cases, of which all eight did not have an indication. Finally, the use of loperamide occurred in one case did not have an indication.

Based on 34 cases, the time patients received their CDI antibiotics after the prescription was written from a physician ranged from ten minutes up to 11 hours, with a mean time of $4: 50 \pm 2: 42$ hours.

\section{Discussion}

The results of this study suggest that CDI treatment adherence to our algorithm has areas for improvement as demonstrated by the $50 \%$ adherence rate measured. The 
disease severity occurring the most at our hospital was mild to moderate, which was fairly well adherent. The treatment regimen consisting of metronidazole $500 \mathrm{mg}$ po q $8 \mathrm{~h}$ for $10-$ 14 days for initial and first recurrences of mild to moderate CDI severity is generally well known. As shown with the breakdown of adherence based on severity, $74 \%$ of the treatment for this disease severity was considered adherent. This treatment is also in agreement with the IDSA 2010 guideline recommendations. However, in cases of severe CDI and severe (Table 2-Table 3) and complicated CDI, IDSA guidelines comment that the classification is not as straight forward and decision depends on clinician expertise and experience. The treatment regimens for severe and severe and complicated recommended by IDSA are consistent with our algorithm - vancomycin $125 \mathrm{mg}$ po q6h and metronidazole $500 \mathrm{mg}$ IV q8h and vancomycin $125-500 \mathrm{mg}$ po q6h, respectively. Further analysis of the breakdown of adherence based on severity demonstrates that the second most common disease severity is severe CDI and this had the highest rate of non-adherence at $83 \%$. Interestingly enough, the signs and symptoms of classification of these disease severity differ, and as a result, the treatment prescribed for each patients may differ depending on which classification of signs and symptoms physicians use. This may be a reason for the low adherence rate as there are some physicians using the IDSA guideline classification instead of the algorithm classification. Lastly, the severe and complicated disease severity is rare at our hospital with only two cases, of which both were non-adherent to treatment.

When analysing the components leading to non-adherence, wrong drug was the highest contributing factor that occurred in $72 \%$ of the cases. The other causes such as wrong dose, route or duration were considered minimal, together comprising a total $28 \%$. This can result as either sub optimal regimens that puts patients at risk of under treatment or increased risk of recurrence. In contrast, treatment with unnecessary antibiotics putting patients at increase risk of adverse effects and excess hospital medication costs depending on the classification. Taking a closer look at the wrong drugs prescribed per disease severity, mild to moderate had a fairly even split of either treatment with vancomycin monotherapy or combination of metronidazole and vancomycin therapy. Both instances are considered unnecessary use of antibiotics as this mild to moderate severity can easily and is well treated with metronidazole monotherapy. In severe disease, which had the highest wrong drug, more than half were prescribed metronidazole monotherapy at $55 \%$ and the rest were prescribed the combination therapy. This highlights an area of focus that under treatment with metronidazole alone may put the patient at increased adverse outcomes associated with CDI. In this severe classification, patients are ill enough to require vancomycin as the antibiotic of choice over metronidazole. Although there are some concerns in prescribing vancomycin to elderly patients with poor renal functions, however because oral vancomycin is not systemically absorbed, this should not be the reason to deter its use. Finally, in severe and complicated, both cases were only treated with metronidazole monotherapy, again putting patients at risk of CDI treatment failures.

Through our study, we identified that the majority of the patients at our hospital who had a CDI were mainly Females age 65 years and over being treated for an initial CDI episode of mild to moderate severity. The two highest risk factors found for our population was the use of at least on antibiotic, most commonly fluoroquinolones and the use of PPIs in the last three months. These findings are consistent with the CDI risk factors identified by IDSA guidelines.

Since there were only seven cases identified that continued CDI antibiotics after non-CDI antibiotics, there were not enough data to accurately describe the current practice of extending therapy at our hospital. The extended duration varied from three days up to two weeks. Furthermore, out of the seven cases of continuation, three had developed a recurrent CDI, which was also not enough data to appropriate describe the rate of recurrent CDI associated with the duration of CDI therapy continuation. Further studies with larger sample size are needed to capture data to answer these two secondary objectives.

Other aspects our study looked at was the use of other medications known to increase the risk of CDI such as concurrent antibiotic use and the ongoing use of PPIs. Not surprisingly, there were about half the patients with CDI on a non-CDI antibiotic and ongoing PPI at the time of diagnosis. However, $96 \%$ of the patients on non-CDI antibiotics had a clear indication, and $19 \%$ of which subsequently had their antibiotics discontinued after reassessing the need for continuation of non-CDI antibiotics. Since many patients are admitted to hospitals for infections, it is common they will be started on antibiotics. This along with other risk factors mentioned in the introduction such as the environment, decreased immune system and exposure to Clostridium difficile bacteria create the ideal host for CDI. At the time of CDI diagnosis, certain infections still need to be treated with the antibiotic that contributed to the CDI. Therefore, weighing the risks vs. benefits, it may be appropriate to continue non-CDI antibiotic and CDI antibiotics concurrently. Regardless of the situation, those non-CDI antibiotics should always be reassessed for indication of continuation depending on the severity, the possibility for cessation where the infection is sufficiently treated or that the infection may not warrant necessary treatment. This was demonstrated with the $19 \%$ antibiotics uses that were subsequently discontinued after CDI diagnosis. PPIs on the other hand had a lower rate of indication at $35 \%$ in the $62 \%$ patients on ongoing PPIs. The use of PPIs is very common for gastrointestinal reflux disease, bleeding ulcers etc. Although there are certain indications that require their use indefinitely, and there is a sufficient number who are initiated on PPIs and remain on them without follow up. With ongoing use, their bodies become dependent on the acid suppression and may develop symptoms of rebound indigestion during abrupt withdrawal.

Fortunately, there are methods such as slowly tapering 
PPIs in patients that do not have a clear indication such as switching to H2RAs or extending PPI regimens to every two days and eventually discontinuing them. The finding that more than a third of patients on PPI whom do not have a clear indication warrant a reassessment of these medications in our patients who develop CDI. This modifiable risk factor becomes a strong reason to consider reassessing PPI use and the possibility of tapering and discontinuing their use.

Other medications assessed were medications recommended to be discontinued at the onset of CDI including laxatives and anti-diarrheal agents. Our retrospective study found that $13 \%$ of ongoing laxatives and $2 \%$ of loperamide were not discontinued in these patients. Whether they were actually given is unclear since we did not have access to all medication administration records (MARs), however points at simple areas of improvement for CDI treatment.

The time to antibiotic use was also measured in this study, and from the 34 cases that identified times of when patients received antibiotics vs. when they were prescribed varied from immediately up to 11 hours. CDI as with other infections is a serious infection and patients should receive their antibiotics as soon as possible, as opposed to waiting for the next scheduled time for antibiotic dosing. The longer time to treatment puts patients at risk of adverse outcomes associated with CDI that can be minimized by prompt antibiotic administration.

Lastly, I looked at overall outcomes of interest to hospitals such as the resolution of CDI, 30-day all cause mortality, 30-day readmission and CDI recurrence. CDI resolution was statistically higher with adherent therapies. However, to note, non-adherent therapies in certain situations resolve CDI similar to adherent therapies. This is the case for mild to moderate CDI, in which treatment considered non-adherent such as vancomycin or dual combination therapy are also effective at resolving CDI. This concept applies to all the other outcomes listed of interest. For example, the 30-day all-cause mortality was also statistically significant lower in patients with adherent therapies. Many times, patients have concomitant infections along with CDI, and whether the cause of death due to a specific infection or in combination with CDI, or for a complete other reason is unknown. Finally, the last two outcomes 30-day readmission and 8 week recurrent CDI were not statistically significant. Therefore, the significance of treatment adherence is less useful in this case for the reasons mentioned above. However, the incidences of these outcomes are of interest. In the CDI resolution, there were $58 \%$ cases that met this outcome. This definition was defined as having 48 hours of no loose stool prior to discharge, and should be kept in mind. Certain patients had concomitant infections and died for other reasons would fall into the category of not resolved CDI. Depending on the severity, signs and symptoms close to discharge, patients do not necessarily need to be kept in hospital. This is the same scenario for 30-day all-cause mortality and 30day readmission, both of which are lower at $23 \%$ and $22 \%$, respectively. Regarding 8-week CDI recurrence, there are other factors besides treatment adherence that impact CDI recurrence such as nonpharmacological environmental cleaning of patient rooms etc. Whether they were reinfected or developed a new CDI either during hospital or after discharge is unknown, and would both count towards 8 -week recurrence.

The strength associated with this study was that the results were relevant to our institution since it can be used for knowledge translation within our institution. In contrast, the limitations associated with this study included it being a retrospective data collection from chart reviews. Therefore, some information were unclear due to the fact that all data were dependent on documentation completed by other health care professionals and were missing. As a result, documentation bias was possible. This was evident in which 26 MARs were missing for 26 CDI cases. Secondly, patients who developed previous or recurrent episode of CDI and were admitted at other institutions were not captured. Thirdly, all information collected and analyzed were limited to the sample size cases limited to the period from June 1, 2017 to June30, 2018.

\section{Conclusion}

In conclusion, an area for improvement in CDI treatment adherence at WDMH is prescribing the right drug based on the disease severity. Specifically in severe CDI, an emphasis is to change therapy to vancomycin alone; in mild to moderate CDI, an emphasis is to change therapy to metronidazole alone. Finally in severe and complicated CDI, an emphasis is to change therapy for dual therapy of metronidazole and vancomycin. PPI use in CDI is a common modifiable risk factor commonly being taken in our population during their CDI. As with all PPI use, and especially a concern in our patients, their use should be reassessed for tapering and discontinuation when appropriate. Lastly, treatment with CDI antibiotics should be prescribed and given to patients as soon as possible after confirmation of CDI diagnosis without any delay.

\section{Acknowledgements}

The author wish to thank The Clinical Information System team (CIS) in helping with generating rep.

\section{References}

[1] Cohen, SH., Gerding, DN, Johnson, S. et al., Clinical Practice Guidelines for Clostridium difficile Infection in Adults: 2010 Update by the Society for Healthcare Epidemiology of America (SHEA) and the Infectious Diseases Society of America (IDSA). Infection Control and Hospital Epidemiology: 2010; 31: 431 - 455.

[2] Public Health Ontario: Monthly Infectious Disease Surveillance Report. Volume 4, Issue 8, Aug 2015. 
[3] Debast, SB. Bauer, MP, Kuijper EJ. European Society of Clinical Microbiology and Infectious Diseases: update of the treatment guidance document for Clostridium difficile infection. Clin Microbiol Infect 2014, 20 (Suppl. 2), 1-26.

[4] Fekety, R., Elmer, G., Greenberg, R., McFarland, L., Mulligan, M., Surawicz, C. Recurrent Clostridium difficile Diarrhea: Characteristics of and Risk Factors for Patients Enrolled in a Prospective, Randomized, Double-Blinded Trial. Clinical Infectious Diseases 1997; 24:324-33.

[5] Owens, R., Donskey C., Gaynes, R., Loo, V., Muto, C. Antibiotic-Associated Risk Factors for Clostridium difficile Infection. Clinical Infectious Diseases 2008; 46:S19-31.

[6] Janarthanan, S., Ditah, I., Douglas, G., M Phil, Murrary, E. Clostridium difficile -Associated Diarrhea and Proton Pump Inhibitor Therapy: A Meta-Analysis. Am J Gastroenterol 2012; 107:1001-1010.

[7] Prévention des Infections. Infection a Clostridium difficile (ICD): Politiques et procedures de Montfort. Fevrier 2016.

[8] Kao, D. Diarrhea. Therapeutics Choices, June 2014.

[9] Pépin J, Valiquette L, Alary ME, Villemure P, Pelletier A, Forget K, Pépin K, Chouinard DClostridium difficileassociated diarrhea in a region of Quebec from 1991 to 2003: a changing pattern of disease severity. CMAJ. 2004; 171(5):466

[10] Vaillancourt, L., Do, K., Shymanski, K, Landry, C. Traitement de l'infection à l'infection clostridium difficile. La capsule Montfort. April 2016.
[11] Surawicz, CM. Brandt, LJ., Binion, DG. Et al. Guidelines for Diagnosis, Treatment, and Prevention of Clostridium difficile Infections. Am J Gastroenterol 2013; 108:478-498.

[12] Health Quality Ontario. Public Reporting. http://www.hqontario.ca/Public-Reporting/PatientSafety/About-Patient-Safety-Public-ReportingTesting, Surveillance and Management of Clostridium difficile In All Health Care Settings: http://www.publichealthontario.ca/en/eRepository/PIDACIPC _Annex_C_Testing_SurveillanceManage_C_difficile_2013.pd $\overline{\mathrm{f}}$

[13] Kelly, CP., Lamont, JT. Clostridium difficile in adults: Treatment. Up-to-date [Internet]. 2017 April [cited 2017 May 14]. Available from: Http: //www.uptodate.com/contents/clostridiumdifficile-in-adultstreatment?source $=$ search_result\&search $=\mathrm{c}+$ difficile\&selected Title $=1 \sim 150$

[14] Gut Sense: Bristol Stool Form Scale: www.gutsense.org356 $\times$ 448Search by image. Retrieved on Aug 1, 2017

[15] McDonald LC, Gerding DN, Johnson S, et al. Clinical practice guidelines for Clostridium difficile infection in adults and children: 2017 update by the Infectious Diseases Society of America (IDSA) and Society for Healthcare Epidemiology of America (SHEA) [published online February 15, 2018]. Clin Infect Dis. doi: 10.1093/cid/cix1085 